Research Article

\title{
Novel splice-affecting variants in CYP27A1 gene in two Chilean patients with Cerebrotendinous Xanthomatosis
}

Susan V. Smalley ${ }^{1 *}$, Yudith Preiss ${ }^{1,2^{*}}$, José Suazo ${ }^{1,3}$, Javier Andrés Vega ${ }^{1}$, Isidora Angellotti ${ }^{1}$, Carlos F. Lagos ${ }^{4}$, Enzo Rivera ${ }^{5,6}$, Karin Kleinsteuber ${ }^{7,8}$, Javier Campion ${ }^{9}$, J. Alfredo Martínez ${ }^{9}$, Alberto Maiz ${ }^{1}$ and José Luis Santos ${ }^{1}$

${ }^{1}$ Department of Nutrition, Diabetes and Metabolism, School of Medicine, Pontificia Universidad Católica de Chile, Santiago, Chile.

${ }^{2}$ School of Medicine, Universidad Diego Portales, Santiago, Chile.

${ }^{3}$ Institute for Research in Dental Sciences, Faculty of Dentistry, Universidad de Chile, Santiago, Chile.

${ }^{4}$ Department of Endocrinology, School of Medicine, Pontificia Universidad Católica de Chile, Santiago, Chile.

${ }^{5}$ Faculty of Medicine, Universidad de Valparaíso, Valparaíso, Chile.

${ }^{6}$ Department of Neurology, Hospital Carlos Van Buren, Valparaiso, Chile.

${ }^{7}$ Faculty of Medicine, Universidad de Chile, Santiago, Chile.

${ }^{8}$ Clínica Las Condes, Santiago, Chile.

${ }^{9}$ Department of Food Sciences and Physiology, Universidad de Navarra, Pamplona, Spain.

\begin{abstract}
Cerebrotendinous Xanthomatosis (CTX), a rare lipid storage disorder, is caused by recessive loss-of-function mutations of the 27-sterol hydroxylase (CYP27A1), producing an alteration of the synthesis of bile acids, with an accumulation of cholestanol. Clinical characteristics include juvenile cataracts, diarrhea, tendon xanthomas, cognitive impairment and other neurological manifestations. Early diagnosis is critical, because treatment with chenodeoxycholic acid may prevent neurological damage. We studied the CYP27A1 gene in two Chilean CTX patients by sequencing its nine exons, exon-intron boundaries, and cDNA from peripheral blood mononuclear cells. Patient 1 is a compound heterozygote for the novel substitution c.256-1G > T that causes exon 2 skipping, leading to a premature stop codon in exon 3, and for the previously-known pathogenic mutation c. 1183C > T (p.Arg395Cys). Patient 2 is homozygous for the novel mutation c.1185-1G > A that causes exon 7 skipping and the generation of a premature stop codon in exon 8, leading to the loss of the crucial adrenoxin binding domain of CYP27A1.
\end{abstract}

Keywords: Cerebrotendinous Xanthomatosis, splicing, mutation, exon skipping.

Received: March 13, 2014; Accepted: October 10, 2014.

\section{Introduction}

Cerebrotendinous xanthomatosis (CTX) [MIM \#213700] is an autosomal recessive disease caused by mutations in the CYP27A1 gene, which encodes the mitochondrial enzyme 27-sterol hydroxylase (EC 1.14.13.15) involved in bile acid synthesis. The primary bile acids cholic acid (CA) and chenodeoxycholic acid (CDCA) are synthesized by two complementary chemical pathways: the classic "neutral pathway", considered as the main producer of bile acids (approximately 50\% CA and CDCA) and an al-

Send correspondence to José Luis Santos. Department of Nutrition, Diabetes and Metabolism, School of Medicine, Pontificia Universidad Católica de Chile, Alameda 340, Santiago, Chile. E-mail: jsantos@med.puc.cl.

*These authors equally contributed to this work. ternative "acidic pathway" leading to CDCA synthesis. The 27-sterol hydroxylase encoded by CYP27A1 catalyzes the initial step in the acidic pathway, with the C27-hydroxylation of cholesterol oxidation of the side chain sterol intermediates in bile acid formation (Sundaram et al., 2008). As a consequence of inherited genomic defects in CYP27A1, there is an alteration of the production of bile acids, mainly decreasing CDCA production. The immediate consequence of this genetic defect is the compensatory increase in the activity of the rate-limiting enzyme in the neutral bile acid synthesis pathway, cholesterol $7 \alpha$-hydroxylase, leading to the accumulation of $7 \alpha$-hydroxylated bile acid precursors, in particular $7 \alpha$-hydroxy-4-cholesten-3-one. This molecule is a precursor of cholestanol, which is elevated in the plasma of CTX patients, as well as an increase in 
plasma cholestanol/cholesterol ratio (Björkhem and Hansson, 2010). Consequently, these molecules are abnormally deposited in different tissues such as central nervous system (CNS) and crystalline lens (Khan et al., 2013).

CTX diagnosis is based on clinical grounds. Recently, an Italian group of experts in CTX has proposed a suspicion index for early diagnosis (Mignarri et al., 2014). Elevated levels of cholestanol levels in serum, high bile alcohol levels in urine and genetic testing, confirm the diagnosis. According to the literature, there is no established genotype-phenotype correlation pattern in CTX. (Lee et al., 2001).

At early stages of CTX, symptoms are childhood diarrhea and bilateral cataracts, which are usually diagnosed during the first or second decade of life (Cenedella, 1996; Moghadasian et al., 2002; Pilo-de-la-Fuente et al., 2011). In more advanced stages, the disease is characterized by tendom xanthomas as well as the presence of progressive neurologic dysfunction signs that usually occur during the second and the third decade of life. Less frequently, the presence of seizures, coronary heart disease and early atherosclerosis has been also described (Dotti et al., 2000).

Although the prevalence of the disease has been roughly estimated in 1/50,000 (Salen et al., 1983), this figure varies by ethnicity and geographic location. In populations of Sephardic Jews of Moroccan origin, a prevalence of 1/108 has been described (Berginer and Abeliovich, 1981), while in Caucasian populations of the United States of America, the estimated prevalence is approximately 3-5/100,000 (Lorincz et al., 2005). There are, so far, more than 50 different CYP27A1 mutations published (http://www.hgmd.org). In Chile, two CTX patients have been previously reported (Filippi et al., 2009; Huijgen et al., 2012). The aim of the present report is to describe the genetic, molecular and clinical characteristics of two Chilean CTX patients.

\section{Subjects and Methods}

\section{Clinical description of patients}

This study was approved by the Ethical Review Board of the School of Medicine of the Pontificia Universidad Católica de Chile. Written informed consent was signed by patients and relatives.

Patient 1: female, 42 years old. No history of consanguinity. History of unexplained chronic diarrhea and bilateral cataracts diagnosed at age of 5 and operated in childhood. Since age of 28 she developed paraparesis and progressive difficulty to walk.Neurological examination in that moment showedbilateral pyramidal syndrome, cerebellar syndrome and deep sensory deficit in lower extremities. Brain magnetic resonance imaging (MRI) showed involutive signs incerebellar and bilateral frontal regions, with white matter involvement bulb and cerebellum. An electrophysiological study of the lower extremities showed signs of chronic sensorymotorpolyneuropathy. Laboratory tests showed negative results for HIV, HTLV-1, and VDRL. Plasma B12 and folic acid levels were normal. Thyroid function was normal. The patient showed a normal lipid profile,exceptfor slightly elevated high density lipoprotein cholesterol (HDL-C) $(74.6 \mathrm{mg} / \mathrm{dL})$. At age of 31, CTX was suspected, although without presenting typical tendinous xanthomas. This patient showed a concentration of $64 \mu \mathrm{mol} / \mathrm{L}$ of plasma cholestanol reported from an external laboratory (normal values: 2-12.6 $\mu \mathrm{mol} / \mathrm{L}$ ). At the moment of recruitment for this study, the patient isprostrated,with a severe spastic tetraparesis, flexor pattern of the four limbs, severe dysphagia that required installation of a percutaneous endoscopic gastrostomy. There is no clinical data of her bone condition at the moment of diagnosis. Due to her clinical condition, it was not possible to perform a bone densitometry at the moment of the recruitment.

Patient 2: male, 17 years old. Patient was adopted at age of 5 months and no data of his biological parents are available. He was hypotonic at infancy, without feeding or respiratory impairments. He had motor and developmental delay, with independent walking after 2.5 years old and language (phrases) after 2 years old. Mother refers frequent stools (twice daily with low consistency by periods) since toddler. Difficulties in visual acuity were noticed at the age of 10 years. Miopic astigmatism and bilateral cataracts were diagnosed at 13 years old. On neurologic examination, the following signs were evident: macrocephaly, dysmorphic signs with mild facial hypomimia, distal hypotrophy in lower limbs; xanthomas on Achilles tendons (both) and one on left triceps tendon. He had speaking, reading, writing and drawing difficulties and distal paresis with hyperreflexia with no spasticity in lower limbs. Fine hands tremor; difficulty in walking on heels and subtle instability in turns were evident. Brain MRI revealed T2 signal abnormalities on cerebellar hemispheric white matter. Thyroid function was normal. Bone densitometry showed low bone mineral mass in both hips and normal spine values (Left femoral neck Z-score -3.3; right femoral neck $Z$-score -3.2). Levels of $25(\mathrm{OH})$ vitamin $D$ were $14.2 \mathrm{ng} / \mathrm{mL}$ (normal values: $20-60 \mathrm{ng} / \mathrm{mL}$ ). Plasma cholestanol levels were analyzed in an external laboratory yielding a result of $2.6 \mathrm{mg} / \mathrm{dL}$ (normal values $0.17 \pm 0.12 \mathrm{mg} / \mathrm{dL}$, for children 3-16 years), thus confirming the diagnosis.

\section{Molecular and bioinformatic methods}

Total DNA and RNA were extracted from peripheral blood in EDTA tubes using Mini blood kits (Qiagen) and Trizol (Ambion) respectively. Genomic DNA was amplified and sequenced (Macrogen, Korea) using primers designed to amplify the 9 intron/exon boundaries, as well as the 3' UTR of the CYP271A1 gene (NG_007959.1). Primer sequences and thermal profiles are available under request from authors. RNA (NM_000784.3) was reverse transcribed with random primers (Applied Biosystem). 
Genomic sequence variation was described according to the guidelines of the Human Genome Variation Society (Den Dunnen and Antonarakis, 2000).

To assess the possible impact of the amino acid substitution p.Arg395Cys on protein structure and function, we used the Polyphen-2 and PROVEAN (v1.1) programs (Adzhubei et al., 2010; Choi et al., 2012). The two novel intronic mutations found in our patients (Table 1) disrupt the canonical splicing 3' acceptor site. Subsequently, we used the Human Splicing finder (HSF) software (Desmet et al., 2009), which predicts the effect of the splice affecting variants with high accuracy based on position weight matrices. Natural splice sites (5'and 3'), branch points and exonic splice enhancers among others are assigned with consensus values. A delta Consensus Value $(\Delta \mathrm{CV})$ of the splice sites strength $>10 \%$ is likely to have an impact on splicing (Desmet et al., 2009). When mutations affecting intron positions $-1,-2,+1$ or +2 occur, the probability of exon skipping, the use of pseudo 3' or 5' splice sites, retention of the mutated intron or cryptic splice sites, among others, is higher (Faustino and Cooper, 2003).

\section{Results}

We found three different mutations in the CYP27A1 gene, listed in Table 1. Patient 1 is a compound heterozygote harboring the novel mutation c. $256-1 \mathrm{G}>\mathrm{T}$ and the previously described missense mutation c.1183 $\mathrm{C}>\mathrm{T}$ (p.Arg395Cys), shown in Figure 1A. Patient 2 is homozygous for the novel mutation c.1185-1G $>$ A, shown in Figure 1B. We have registered the new CYP27A1 mutations in the Leiden Open Variation Database with accession numbers as following c.256-1G $>\mathrm{T}$ : \# DB_ID:CYP27A1_00069, and c-1185-1G > A: \# DB ID:CYP27A1 00068. The third mutation c.1183C > T or p.Arg395Cys (patient 1) has accession number: \# DB_ID:CYP27A1_00004 (http://www.lovd.nl/3.0/home), is shown in Figure 1A.

The analysis of the novel mutation c.256-1G $>\mathrm{T}$ on the splicing process, using the HSF program, predicted that the nucleotide $\mathrm{T}$ lowers the strength $(\Delta \mathrm{CV}-32.9 \%)$ of the natural splicing acceptor 3' site (AG $>A T)$ and also seems to swop the 3' site to a 5' donor site with a $\triangle \mathrm{CV}-14.0$. This would predict a highly plausible skipping of exon 2 (191 nucleotides long). This imprecise splicing may shift the reading frame creating a new amino acid sequence of 30 residues containing a premature stop codon (GRTPLVPAAPGSEPAVAEASGSSALYGCFQ*).

In order to demonstrate the effect of the mutations found in patient 1 , we reverse transcribed total RNA extracted from leukocytes of peripheral blood. The cDNA was amplified and sequenced in two segments: exon 1 to 4 , and exon 5 to 9 . The sequence was concordant with the compound heterozygote genotype found in the genomic DNA, as both mutations were present. The cDNA sequence

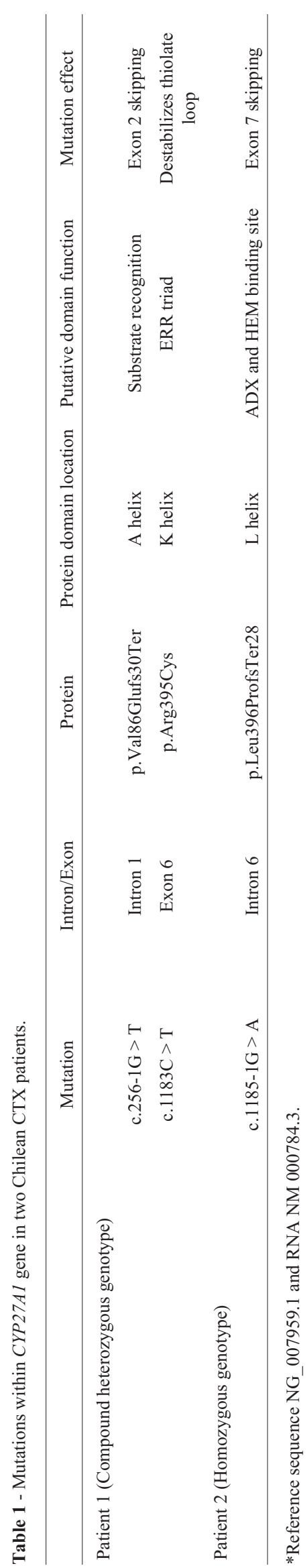


A)

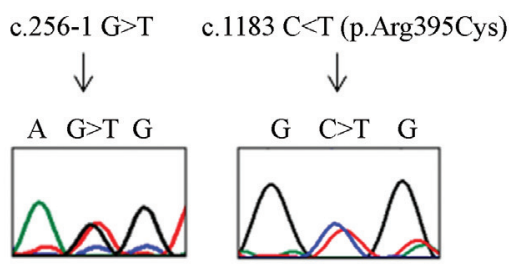

B)

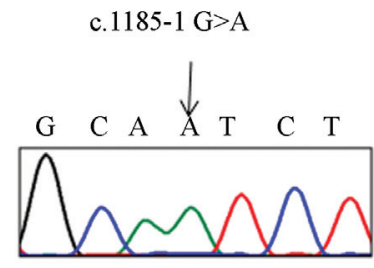

C)

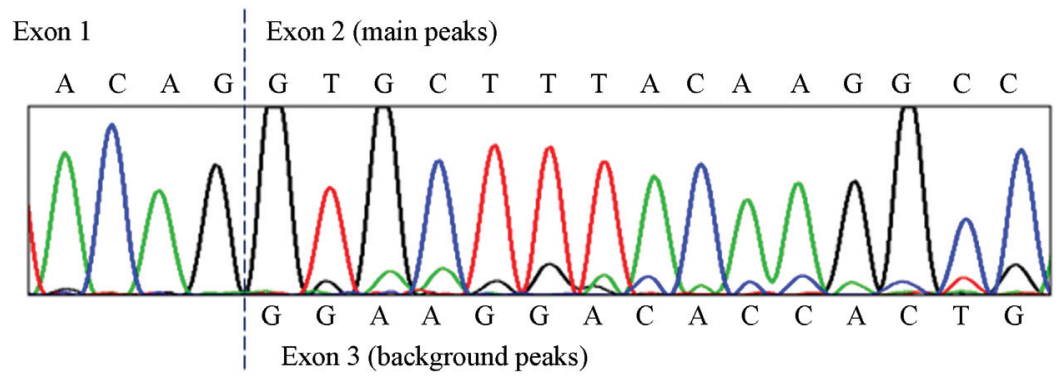

D)

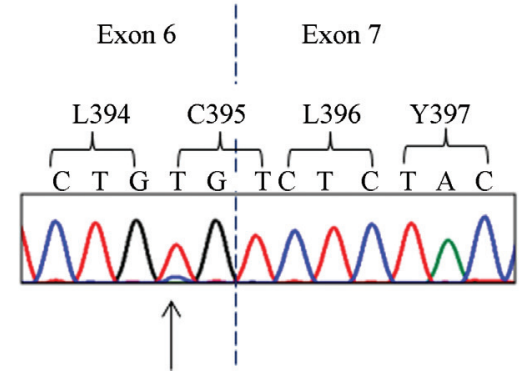

E)

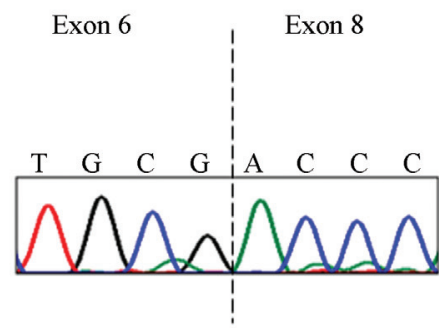

Figure 1 - Genomic and cDNA sequences showing case 1 and case 2 mutations. A) Case 1 genomic sequence showing heterozygote mutations. Black arrows point to mutated nucleotides. B) Case 2 genomic sequence. Homozygous mutation shown by arrow. C) cDNA sequence of case 1 (Exons 1-3). One haplotype has the normal sequence of exon 1 joined to exon 2, while the second haplotype shows exon 1 incorrectly joined to exon 3 (small peaks) (RefSeq NG_007959). D) cDNA sequence of case 1 showing exon 6 and 7 with the mutation at codon 395. E) cDNA sequence of case 2 showing the join of exon 6 to exon 8 , skipping exon 7.

of exon 1 to 4 of showed the extended haplotype sequence containing the intronic mutation c.256-1G $>$ T and the normal haplotype. The main peaks correspond to the nucleotide sequence of exon 2, while the weak background peaks of the sequence correspond to the nucleotide sequence of exon 3 (Figure 1C). This result indicates that exon 2 is incorrectly spliced and exon 1 is joined to exon 3 in the extended haplotype carrying the c.256-1G $>\mathrm{T}$ mutation. Thus, we have demonstrated the existence of exon skipping, which was previously predicted by the HSF program. The mutation c.256-1G $>\mathrm{T}$ has been named as p.Val86Glyfs30Ter (Table 1).

Patient 1 cDNA also carry the c.1183C $>\mathrm{T}$ (p.Arg395Cys) variant, which is located in the cDNA sequence corresponding to a TGT codon, codifying for cysteine, as shown in Figure 1D. The first two nucleotides of this codon come from exon 6 and the third nucleotide comes from exon 7 , with the c. $1183 \mathrm{C}>\mathrm{T}$ mutation thus being located in the penultimate site of exon 6. The cDNA sequence analysis showed that there is no splice defect derived from this mutation, given that exon 6 is correctly joined with exon 7. On the other hand, the c.1183C $>$ T mutation generates a change in the amino acid sequence (p.Arg395Cys) previously known to be pathogenic. According to bioinformatics predictions, Polyphen-2 showed that p.Arg395Cys was "probably damaging" with the highest score possible of 1.0 (range 0.0 to 1.0). The tridimensional model of CYP27A1 $( \pm \mathrm{A})$ shows that Arg395 interacts with Phe 438 by pi-cationic interaction and is also involved in h-bond interactions with the carboxyl group of Glu392 and the main-chain nitrogen from Pro441 (Figure 2B upper panel).

We found the novel mutation c.1185-1G $>\mathrm{A}$ in the "-1" site of intron 6 in homozygous state in patient 2 (Figure 1B). The analysis of this mutation using HSF software showed a decrease in the strength of the 3' splice site $(\triangle \mathrm{CV}$ $-30.7 \%$ ) and MaxEnt revealed a $\triangle \mathrm{CV}$ of $-119.7 \%$ in strength variation. Both values can be considered strong evidence to predict a splice defect. Following the same procedure used in Patient 1, we reverse transcribed total RNA and amplified and sequenced the cDNA from patient 2. The cDNA sequence of exon 5 to exon 9 of CYP27A1 of patient 


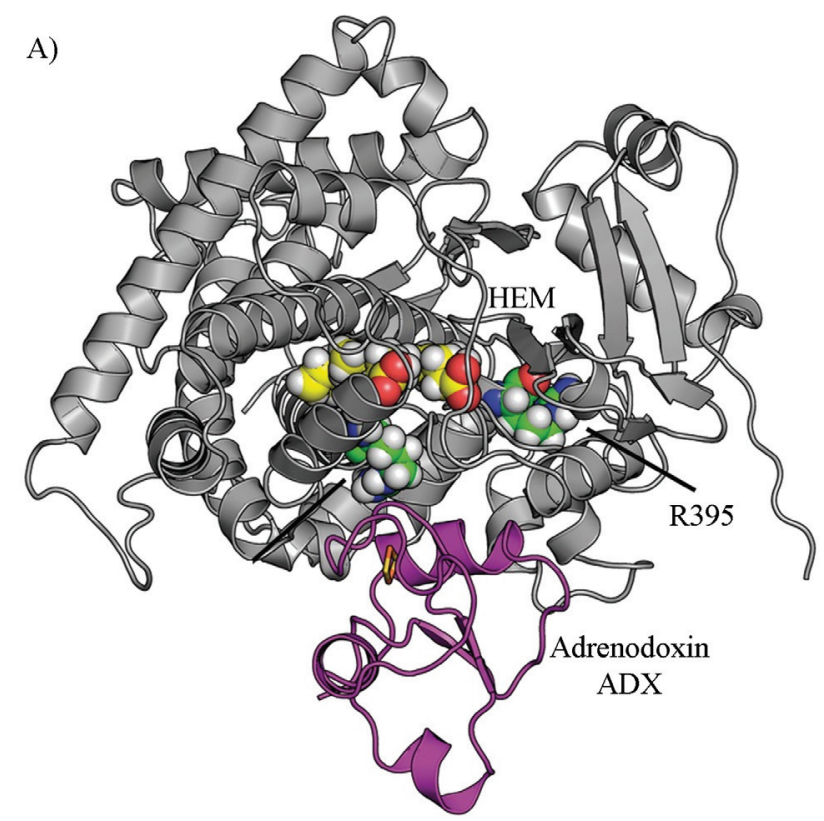

B)
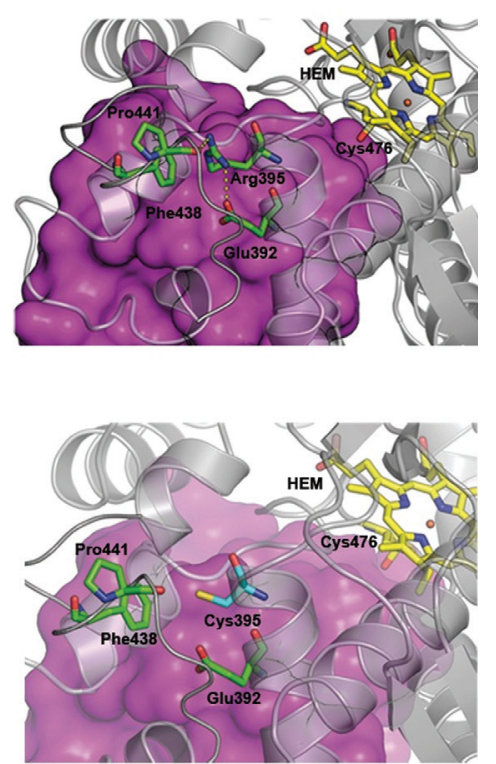

Figure 2 - CYP27A1 modeling. A) Tridimensional model of wild type protein CYP27A1 showing HEM group and position of Arg395. B (upper panel): Enlargement of amino acids region showing that Arg395 interacts with Phe 438 by pi-cationic interaction and is also involved in $\mathrm{H}$-bond interactions with the carboxyl group of Glu392 and the main-chain nitrogen from Pro441. B (lower panel): Mutation to Cys395 will increase distances and the interactions loosened will favor misfolding, which may affect ADX binding.

2 shows that exon 6 is joined to exon 8 (Figure 1E), demonstrating that the homozygous mutation did affect normal splicing of exon 7. We expect that the mutated protein loses 136 amino acids of the original sequence and only preserves sequence identity up to $\operatorname{Arg} 395$, after this position there is a new sequence of 28 amino acids (PSLCSATMWCPGTPLPSLSLKASSPTAG*), which finalizes with a premature stop codon, generating a predicted truncated protein of 423 amino acids. The nomenclature for this mutation is p.Leu396ProfsTer28 (Table 1).

\section{Discussion}

As a consequence of the metabolic impairments observed in Cerebrotendinous xanthomatosis, it has been proposed that the primary treatment of this disease would be oral CDCA administration, which is reported to decrease plasma cholestanol levels (Sundaram et al., 2008). Statins may also produce a synergistic effect by lowering the cholesterol pool and improving the clinical response (Watts et al., 1996; Verrips et al., 1999; Federico and Dotti, 2003).

Patient 1 is a compound heterozygote for two CYP27A1 variants: c.256-1G $>\mathrm{T}$ and the missense mutation c. $1183 \mathrm{C}>\mathrm{T}$ (p.Arg395Cys). The c.256-1G $>$ T mutation has not been previously reported in other case series. We show here molecular evidence based on cDNA analysis that such a mutation leads to a splice defect, also supported by bioinformatics analysis. In this context, the c.256-1G > $\mathrm{T}$ mutation causes exon 2 skipping and a premature stop codon in exon 3 (p.Val86Glyfs30Ter). The second mutation found in patient $1(\mathrm{c} .1183 \mathrm{C}>\mathrm{T})$, alters the protein se- quence (p.Arg395Cys). This mutation (also termed p.Arg362Cys) is the most common mutation found in CTX patients of Spanish origin (Pilo de la Fuente et al., 2011). It is very likely that its presence in Chile is derived from migrations from Spain, one of the main population contributors to the current Chilean population. It was shown by Cali et al. (1991) that the p.Arg395Cys mutation dramatically reduces enzyme activity. In our study, we provide support to this observation using bioinformatics tools. According to our tridimensional protein model, position 395 is located within the ERR triad, which may act as a folding motif, stabilizing heme binding and redox partner binding (Prosser $e t$ al., 2006). It is predicted that mutation in Cys 395 may increase distances and the loosened interactions may favor misfolding, thus affecting adrenoxin (ADX) binding (Figure 2B lower panel).

It is worthy of note that the mutation c.Arg395Ser is located in the same genomic position (penultimate nucleotide of exon 6) as the c.Arg395Cys (codon CGT to TGT; c.1183C > T) (Chen et al. (1998). Given the special location of this mutation in the exon-intron boundary, Chen et al. (1998) proposed that the c.Arg395Ser mutation may affect normal splicing and gene expression efficiency, implying that this specific genomic DNA position may also affect gene expression in other mutations located in the same genomic site. As far as we know, there are no reports assessing the possible impact of the mutation c. $1183 \mathrm{C}>\mathrm{T}$ on gene expression or the splicing process. Although we have not determined whether c. $1183 \mathrm{C}>\mathrm{T}$ affects gene expression efficiency, we show here that the sequence containing the c.1183C $>$ T mutation is fully transcribed (Figure 1D). 
Therefore, it is very likely that the sole effect of the amino acid change generated by the amino acid change c.Arg395Cys is sufficient to impair enzyme activity and cause CTX (Cali et al., 1991).

Regarding the c.1185-1G > A mutation found in homozygous state in patient 2 , we also show bioinformatic and molecular evidence supporting a role in the CYP27AI splicing process. We show that this mutation causes exon 7 skipping and the generation of a premature stop codon in exon 8 . As a consequence, the protein lacks the two binding domains for its cofactors adrenodoxin (ADX) and ADX reductase, as well as relevant residues for HEM coordination such as Cys476. It is also possible that this patient lacks the CYP27A1 protein, as the stop codon generated by exon skipping is located $>55$ nucleotides upstream of the most 3 ' exon-exon junction, possibly leading the activation of the Nonsense-Mediated mRNA Decay (NMD) surveillance pathway (Karam et al., 2013).

It is necessary to emphasize the importance of clinical and biochemical CTX screening in young patients with early disease signs, such as neonatal cholestatic jaundice, childhood diarrhea, and congenital or juvenile bilateral cataracts, given that a treatment with CDCA is available. In the absence of national screening programs, ophthalmological findings in patients with CTX are interesting since they are one of the first signs of the disease, often before diagnosis of CTX is made. It is important to emphasize that early treatment during childhood and before CNS deposits appear, may avoid progression of the disease.

In conclusion, we have reported herein the genetic, molecular and clinical characteristics of two Chilean CTX cases, showing two novel splice-affecting variants in the CYP27A1 gene. Since CTX is a treatable disease, we propose to screen new CTX cases among children with early disease signs, such as bilateral cataracts, followed by testing their plasma cholestanol levels and searching mutations in the CYP27A1 gene in the patients who are positive for biochemical screening.

\section{Acknowledgments}

Supported by Dirección de Investigación (School of Medicine, Pontificia Universidad Católica de Chile) and the Department of Nutrition, Diabetes and Metabolism (School of Medicine, Pontificia Universidad Católica de Chile). We would like to thank patients and their parents for their collaboration.

\section{References}

Adzhubei IA, Schmidt S, Peshkin L, Ramensky VE, Gerasimova A, Bork P, Kondrashov AS and Sunyaev SR (2010) A method and server for predicting damaging missense mutations. Nat Methods 7:248-249.

Berginer VM and Abeliovich D (1981) Genetics of cerebrotendinous xanthomatosis (CTX) an autosomal recessive trait with high gene frequency in sephardic of moroccan origin. Am J Med Genet 10:151-157.

Björkhem I and Hansson M (2010) Cerebrotendinous xanthomatosis: An inborn error in bile acid synthesis with defined mutations but still a challenge. Biochem Biophys Res Commun 396:46-49.

Cali JJ, Hsieh CL, Francke U and Russell DW (1991) Mutations in the bile acid biosynthetic enzyme sterol 27-hydroxylase underlie cerebrotendinous xanthomatosis. J Biol Chem 266:7779-7783.

Cenedella RJ (1996) Cholesterol and cataracts. Surv Ophthalmol 40:320-337.

Chen W, Kubota S, Ujike H, Ishihara T and Seyama Y (1998) A novel Arg362Ser mutation in the sterol 27-hydroxylase gene (CYP27): Its effects on pre-mRNA splicing and enzyme activity. Biochemistry 37:15050-15056.

Choi Y, Sims GE, Murphy S, Miller JR and Chan AP (2012) Predicting the functional effect of amino acid substitutions and indels. PLoS One 7:e46688.

Den Dunnen JT and Antonarakis SE (2000) Mutation nomenclature extensions and suggestions to describe complex mutations: A discussion. Hum Mutat 15:7-12.

Desmet FO, Hamroun D, Lalande M, Collod-Beroud G, Claustres M and Beroud C (2009) Human Splicing Finder: An online bioinformatics tool to predict splicing signals. Nucleic Acid Res 37:e67.

Dotti MT, Federico A, Garuti R and Calandra S (2000) Cerebrotendinous xanthomatosis with predominant Parkinsonian syndrome: Further confirmation of the clinical heterogeneity. Mov Disord 15:1017-1019.

Faustino NA and Cooper TA (2003) Pre-mRNA splicing and human disease. Genes Dev 17:419-437.

Filippi J, Irarrázaval S, Peredo P and Mellado P (2009) Cerebrotendinous xanthomatosis: Report of one case. Rev Med Chile 137:815-820.

Huijgen R, Stork AD, Defesche JC, Peter J, Alonso R, Cuevas A, Kastelein JJ, Duran M and Stroes ES (2012) Extreme xanthomatosis in patients with both familial hypercholesterolemia and cerebrotendinous xanthomatosis. Clin Genet 81:24-28.

Karam R, Wengrod J, Gardner LB and Wilkinson MF (2013) Regulation of nonsense-mediated mRNA decay: Implications for physiology and disease. Biochim Biophys Acta 1829:624-633.

Khan AO, Aldahmesh MA, Mohamed JY and Alkuraya FS (2013) Juvenile cataract morphology in 3 siblings not yet diagnosed with cerebrotendinous xanthomatosis. Ophthalmology 120:956-960.

Lee MH, Hazard S, Carpten JD, Yi S, Cohen J, Gerhardt GT, Salen G and Patel SB (2001) Fine-mapping, mutation analyses, and structural mapping of cerebrotendinous xanthomatosis in U.S. pedigrees. J Lipid Res 42:159-169.

Lorincz MT, Rainier S, Thomas D and Fink JK (2005) Cerebrotendinous xanthomatosis: Possible higher prevalence than previously recognized. Arch Neurol 62:1459-1463.

Mignarri A, Gallus GN, Dotti MT and Federico A (2014) A suspicion index for early diagnosis and treatment of cerebrotendinous xanthomatosis. J Inherit Metab Dis 37:421-429.

Moghadasian MH, Salen G, Frohlich JJ and Scudamore CH (2002) Cerebrotendinous xanthomatosis: A rare disease with diverse manifestations. Arch Neurol 59:527-529. 
Pilo-de-la-Fuente B, Jimenez-Escrig A, Lorenzo JR, Pardo J, Arias M, Ares-Luque A, Duarte J, Muñiz-Pérez S and Sobrido MJ (2011) Cerebrotendinous xanthomatosis in Spain: Clinical, prognostic, and genetic survey. Eur J Neurol 18:1203-1211.

Prosser DE, Guo Y, Jia Z and Jones G (2006) Structural motif-based homology modeling of CYP27A1 and site-directed mutational analyses affecting vitamin D hydroxylation. Biophys J 90:3389-3409.

Salen G, Shefer S and Berginer VM (1983) Familial diseases with storage of sterols other than cholesterol: Cerebrotendinous xanthomatosis and sitosterolemia with xanthomatosis. In: Stanbury JB, Wyngaarden JB, Fredrickson DS, Goldstein JL and Brown MS (eds) The Metabolic Basis of Inherited Disease. $5^{\text {th }}$ edition. McGraw-Hill, New York, pp 713-730.
Sundaram SS, Bove KE, Lovell MA and Sokol RJ (2008) Mechanisms of disease: Inborn errors of bile acid synthesis. Nat Clin Pract Gastroenterol Hepatol 5:456-468.

Verrips A, Wevers RA, Van Engelen BG, Keyser A, Wolthers BG, Barkhof F, Stalenhoef A, De Graaf R, Janssen-Zijlstra F, Van Spreeken A, et al. (1999) Effect of simvastatin in addition to chenodeoxycholic acid in patients with cerebrotendinous xanthomatosis. Metabolism 48:233-238.

Watts GF, Mitchell WD, Bending JJ, Reshef A and Leitersdorf E (1996) Cerebrotendinous xanthomatosis: A family study of sterol 27-hydroxylase mutations and pharmacotherapy. QJM 89:55-63.

Associate Editor: Mara H. Hutz

License information: This is an open-access article distributed under the terms of the Creative Commons Attribution License, which permits unrestricted use, distribution, and reproduction in any medium, provided the original work is properly cited. 